\title{
2020 JGS Best Paper Award and the Editors' Choice Paper Volume 23(1)
}

\author{
Manfred M. Fischer ${ }^{1}$ (D) Antonio Paez ${ }^{2}$ (D) Andrés Rodríguez-Pose ${ }^{3}$ (D) \\ Petra Staufer-Steinnocher ${ }^{1}$ D
}

Received: 16 February 2021 / Accepted: 16 February 2021 / Published online: 4 March 2021

(C) The Author(s), under exclusive licence to Springer-Verlag GmbH, DE part of Springer Nature 2021

With the first issue of 2021, it is our pleasure to announce two novel initiatives to acknowledge and celebrate the outstanding quality of research published in the journal: first, the new annual JGS Best Paper Award, and second, the Editors' Choice of their favorite paper of each issue. The members of the editorial board of the journal and/or the editors may nominate candidates for the JGS Best Paper award. Following the nominations, the decision about the award rests with the editors-in-chief of the journal. The winners of the yearly award and the authors of the quarterly editors' choice articles will be listed on the website of the journal, alongside previous awardees. Their respective papers will be made free access for a certain amount of time.

The objective of the JGS Best Paper Award is to encourage and recognize excellent scholarship published in the journal in the preceding year. For the year 2020, two truly excellent papers stand out. They properly represent the broad scope of a journal that covers the fields of GIScience and spatial planning, as well as spatial statistics and econometrics.

In 2020 JGS published 22 papers, all undergoing a stringent peer review process. All of them are of excellent quality, making any decision about prizes a difficult one. Choosing among this pool of top research in our field was difficult. But a decision had to be made and it is our great pleasure to announce that the joint winners of the 2020 JGS Best Paper Award are the contributions by James P LeSage and by Scott A Hemmerling, Monica Barra, Harris C. Bienn, Melissa M. Baustian, Hoonshin Jung, Ehab Meselhe, Yushi Wang, and Eric White. Their respective original research papers are summarized next and can be accessed in full via the Journal's homepage (DOI and read-only links are given below).

Petra Staufer-Steinnocher

petra.staufer-steinnocher@wu.ac.at

1 WU Vienna University of Economics and Business, Vienna, Austria

2 McMaster University, Hamilton, Ontario, Canada

3 London School of Economics, London, United Kingdom 


\section{Fast MCMC estimation of multiple $\mathrm{W}$-matrix spatial regression models and Metropolis-Hastings Monte Carlo log-marginal likelihoods}

by James P. LeSage, published in JGS 22 (2020), pages 47-75 (https://doi.org/10. 1007/s10109-019-00294-2; https://rdcu.be/ceypz)

This paper describes an approach for rapid Markov Chain Monte Carlo (MCMC) estimation of a general family of spatial econometric models that may contain multiple simultaneous dependence parameters. Such parameters can complicate both maximum likelihood and MCMC estimation. Computational challenges to estimating these models arise from two issues. The first relates to the need to evaluate the log-determinant term that arises in the log-likelihood for multiple dependence parameters, and to maximize a multi-parameter likelihood. And the second concerns the need to impose stability restrictions that involve the multiple dependence parameters. The paper outlines newly minted estimation procedures and techniques to tackle these challenges for the case of massive data samples. An empirical application illustrates some of the advantages of the MCMC estimation approach, using convex combination of weight matrices models. These advantages also apply to dynamic space-time panel models and gravity models of origin-destination flows that are specific cases of the general model family. The publication of this paper presents a landmark that induces an important step forward in the state of the art of applied spatial econometrics. The abstract of the paper is as follows:

A strategy for MCMC estimation of a family of models involving multiple simultaneous dependence parameters is set forth that is capable of producing rapid estimates for problems involving a large number of observations. Simultaneous dependence parameters arise when dependence exists between dependent variable observations, with spatial and cross-sectional dependence being specific examples. The approach taken is to express the joint conditional distribution of the dependence parameters as a quadratic form, where the dependence parameters are outer vectors of the quadratic form and the inner matrix expressions of the quadratic form involve only sample data that allow these to be pre-calculated. During MCMC estimation, multiple evaluations of the joint conditional distribution of the dependence parameters can be carried out rapidly, allowing a block-sampling scheme. Block sampling of the dependence parameters is useful for imposing stability restrictions that arise for these parameters. In addition, a Taylor series approximation to the log-determinant expression that arises in the joint conditional distribution for the dependence parameters can be used to rapidly evaluate this term. The joint conditional distribution for the dependence parameters is obtained by analytically integrating out other model parameters, allowing Monte Carlo integration of the log-marginal likelihood, which can be used for model comparison. Timing results are discussed along with 
Monte Carlo evidence regarding performance as well as applied illustrations involving large sample sizes.

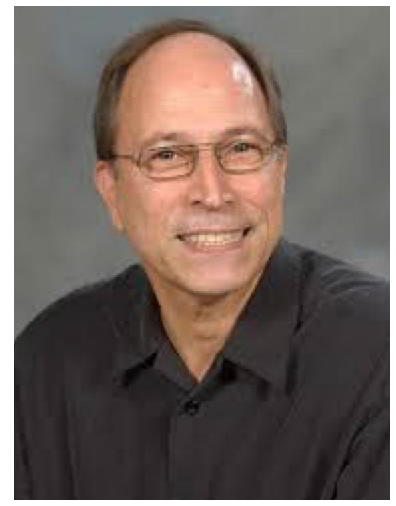

(Source: https://faculty.txstate.edu/profile/520670)

James LeSage received his $\mathrm{PhD}$ in economics from Boston College and has held faculty positions at Bowling Green State University and University of Toledo. Since 2006, he has been the Fields Endowed Chair in Urban \& Regional Economics at Texas State University. He is a fellow of the Regional Science Association International, Spatial Econometrics Association and Southern Regional Science Association, and in 2019 was given the Jean Paelinck Award for Distinguished Sustained Scholarship in Regional Science Methods by the Regional Science Association International. He has published over 100 scholarly journal articles, and is co-author with R. Kelley Pace of a 2009 book entitled Introduction to Spatial Econometrics that book has received over 5000 citations. His research has received past support from the National Science Foundation, and he has given workshops on spatial econometrics in Austria, China, France, Portugal, Spain, and several American universities.

On behalf of the editors-in-chief and the editorial team of Journal of Geographical Systems, our warmest congratulations to the inaugural awardee of the JGS Best Paper Award!

\section{Elevating local knowledge through participatory modeling: active community engagement in restoration planning in coastal Louisiana}

by Scott A. Hemmerling, Monica Barra, Harris C. Bienn, Melissa M. Baustian, Hoonshin Jung, Ehab Meselhe, Yushi Wang \& Eric White, published in JGS 22 (2020), pages 241-266 (https://doi.org/10.1007/s10109-019-00313-2; https://rdcu. be/ceyqm) 
The paper describes a multidisciplinary team of modelers and their work with stakeholders to integrate local knowledge into their modeling practice. The result of this effort is not only better science, but greater legitimacy of the output and better chances of buy-in by the stakeholders. At a time when objective fact is relentlessly under attack for political purposes, approaches to involve multiple stakeholders as part of the scientific process are valuable for education, policy, and to increase the acceptability/acceptance of science. The abstract of the paper is as follows:

Numerical modeling efforts in support of restoration and protection activities in coastal Louisiana have traditionally been conducted externally to any stakeholder engagement processes. This separation has resulted in planning- and project-level models built solely on technical observation and analysis of natural processes. Despite its scientific rigor, this process often fails to account for the knowledge, values, and experiences of local stakeholders that often contextualizes a modeled system. To bridge this gap, a team of natural and social scientists worked directly with local residents and resource users to develop a participatory modeling approach to collect and utilize local knowledge about the Breton Sound Estuary in southeast Louisiana, USA. Knowledge capture was facilitated through application of a local knowledge mapping methodology designed to catalog local understanding of current and historical conditions within the estuary and identify desired ecological and hydrologic end states. The results of the mapping endeavor informed modeling activities designed to assess the applicability of the identified restoration solutions. This effort was aimed at increasing stakeholder buy-in surrounding the utility of numerical models for planning and designing coastal protection and restoration projects and included an ancillary outcome aimed at elevating stakeholder empowerment regarding the design of nature-based restoration solutions and modeling scenarios. This intersection of traditional science and modeling activities with the collection and analysis of traditional ecological knowledge proved useful in elevating the confidence that community members had in modeled restoration outcomes. 


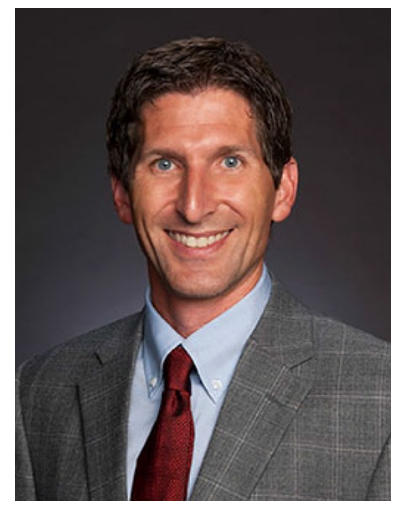

(Source: https://thewaterinstitute.org/our-team/scott-hemmerling)

Scott A. Hemmerling is the Director of Human Dimensions for The Water Institute of the Gulf (Baton Rouge, LA, USA) focusing on research related to climate adaptation and community resilience. He has more than fifteen years of experience investigating anthropogenic alterations to the landscape and the impacts of development on coastal communities. His recent work with the Water Institute is focused on analyzing the societal impacts of environmental change in coastal Louisiana and developing methods to examine the linkages between human and natural systems. Scott earned a PhD in Geography and Anthropology from Louisiana State University: Baton Rouge, LA, USA.

Monica Barra is an assistant professor in the area of Race and Environment at the School of the Earth, Ocean, and Environment and Department of Anthropology and is a faculty affiliate in African American Studies at the University of South Carolina, Columbia, SC, USA. She holds a Ph.D. in Cultural Anthropology from the Graduate Center at the City University of New York.

Harris C. Bienn, Melissa M. Baustian, Hoonshin Jung, Yushi Wang \& Eric White are or were working with the Water Institute of the Gulf, Baton Rouge, LA, 70802, USA.

Ehab Meselhe is Professor in the Department of River-Coastal Science and Engineering at Tulane University. Dr. Meselhe has more than 25 years of experience researching coastal wetland hydrology, sediment transport, and computer modeling of coastal wetland, estuarine, and riverine systems. He also served as an Associate Editor of the Journal of Hydrology (Elsevier), and the Journal of Hydraulic Research (International Association of Hydraulic Research). 
On behalf of the editors-in-chief and the editorial team of Journal of Geographical Systems, our warmest congratulations to the inaugural awardees of the JGS Best Paper Award!

Editors' Choice Paper Volume 23(1)

The JGS Editors' Choice of articles represent papers that the editors see as providing an especially significant contribution to the field. The first JGS Editors' Choice from the current volume 23(1) could not be timelier in this beginning second year of the pandemic. The authors document the relationship between relative risk of COVID-19 and mortality, inequality as well as socioeconomic vulnerability variables. As one of the anonymous reviewers stated, two relevant issues are pointed out in this original contribution: firstly, the analysis of COVID-19 incidence at a municipal level, which is, to our knowledge, the first in Brazil at such low level of aggregate; secondly, the use of Scan statistics to identify 'live' clusters which is mainly useful for the identification of cluster alarms. The spatiotemporal scan test is one of the most popular methods among public health officials and researchers.

Detecting space-time clusters of COVID-19 in Brazil: mortality, inequality, socioeconomic vulnerability, and the relative risk of the disease in Brazilian municipalities

by Marcos Roberto Martines, Ricardo V Ferreira, Rogério Hartung Toppa, Luiza Maria de Assunção, Michael R Desjardins \& Eric M Delmelle (https://doi.org/10. 1007/s10109-020-00344-0)

On behalf of the editors-in-chief and the editorial team of Journal of Geographical Systems, our warmest congratulations to the inaugural awardees of the JGS Editors' Choice Award!

Publisher's Note Springer Nature remains neutral with regard to jurisdictional claims in published maps and institutional affiliations. 